\title{
Planar of special idealization rings
}

\author{
MANAL Al-LABADI \\ Eman Mohammad Almuhur \\ Department of Mathematics \\ University Of Petra \\ Amman, JORDAN \\ Department of Mathematics, \\ Faculty of Basic Sciences and Humanities, \\ Applied Science Private University, \\ Amman, JORDAN
}

Abstract: Let $\mathbf{R}(+) N$ be the idealization of the ring $\mathbf{R}$ by the $\mathbf{R}$-module $N$. In this paper, we investigate when $\Gamma(\mathbf{R}(+) \mathbf{N})$ is a Planar graph where $\mathbf{R}$ is an integral domain and we investigate when $\Gamma\left(\mathbf{Z}_{n}(+) \mathbf{Z}_{m}\right)$ is a Planar graph.

Key-Words: The idealization rings R, Planar graph, Zero-divisor graph.

Received: July 27, 2020. Revised: November 23, 2020. Accepted: December 10, 2020. Published: December 23, 2020.

\section{Introduction}

I. Beck in [6] introduce the concept of zero-divisor graph also, D. D. Anderson and M. Naseer in [3] studied the context of coloring which is an interest concept of graph theory. Anderson and Livingston in $[4$, Theorem 2.3] give the definition of the zerodivisor graph. For more information in zero-divisor graph see, [5].

Let $\mathbf{R}$ be a commutative ring, the zero-divisor graph is the graph $\Gamma(\mathbf{R})$ which vertices are the non-zero zero divisors of $\mathbf{R}$, with $a$ and $b$ adjacent if $a=b$ and $a . b=0$.

For each ring $\mathbf{R}$, the set of all zero-divisors of the ring $\mathbf{R}$ is $Z(\mathbf{R})$.

The idealization ring $\mathbf{R}(+) N$ is defined as $\mathbf{N}$ be an R-module and let $\mathbf{R}(+) \mathbf{N}=\left\{\left(a_{1}, h_{1}\right)\right.$ : $\left.a_{1} \in \mathbf{R}, h_{1} \in \mathbf{N}\right\}$ we have two operations $\left(a_{1}, h_{1}\right)+\left(a_{2}, h_{2}\right)=\left(a_{1}+a_{2}, h_{1}+h_{2}\right)$ and $\left(a_{1}, h_{1}\right)\left(a_{2}, h_{2}\right)=\left(a_{1} a_{2}, a 1 h_{2}+a_{2} h_{1}\right)$.

Another concept of interest in the graph theory. The Planar graph is a graph isomorphic to a Plane graph. A Plane graph is graph that can be drawn on the plane without cross edging. If the graph has induced subgraph isomorphic to $K_{5}$ that is not a Planar graph, by Kuratoskies Theorem.

\section{When $\Gamma(\mathbf{R}(+) \mathbf{N})$ is a Planar graph?}

In this section, we investigate when $\Gamma(\mathbf{R}(+) \mathbf{N})$ is Planar graph where $\mathbf{R}$ is an integral domain and $\mathbf{N}$ be an R-module.

We begin with the following lemma when $\mathbf{R}$ is an integral domain for the idealization ring $\mathbf{R}(+) \mathbf{N}$.

\section{Lemma 1:}

[2] Suppose that $\mathbf{R}$ is an integral domain and $\mathbf{N}$ is an $\mathbf{R}$-module. Then we have the following cases:

- Case 1. If $\mathbf{R}$ is an integral domain with $\mathbf{N} \cong \mathbf{Z}_{2}$ is an $\mathbf{R}$-module and annihilator of $\mathbf{Z}_{2}$ is equal to zero, then the integral domain $\mathbf{R}$ is $\mathbf{R} \cong \mathbf{Z}_{2}$.

- Case 2. If $\mathbf{R}$ be an integral domain with $\mathbf{N} \cong \mathbf{Z}_{3}$ is an $\mathbf{R}$-module and annihilator of $\mathbf{Z}_{3}$ is equal to zero, then the integral domain $\mathbf{R}$ is $\mathbf{R} \cong \mathbf{Z}_{3}$.

\section{Theorem 1:}

Suppose that $\mathbf{R}$ is an integral domain and $\mathbf{N} \cong \mathbf{Z}_{2}$ is an $\mathbf{R}$-module. Then the graph $\Gamma\left(\mathbf{R}(+) \mathbf{Z}_{2}\right)$ is a Planar.

Proof:

To proof we have the following two cases to thoughtfulness:

- Case 1: If the annihilator of $\mathbf{Z}_{2}$ is equal to zero, then $\Gamma\left(\mathbf{Z}_{2}(+) \mathbf{Z}_{2}\right)$ is equal to $\{(0,1)\}$ which is a Planar graph.

- Case 2: If the annihilator of $\mathbf{Z}_{2}$ is not equal to zero, then the graph $\Gamma\left(\mathbf{R}(+) \mathbf{Z}_{2}\right)=$ $\left\{(0,1),\left(k_{i}, 0\right),\left(k_{j}, 1\right): k_{i}, k_{j} \in \operatorname{ann}\left(\mathbf{Z}_{2}\right)\right\}$. So, the graph $\Gamma\left(\mathbf{R}(+) \mathbf{Z}_{2}\right)$ is a star which is a Planar graph.

Theorem 2:

Suppose that $\mathbf{R}$ is an integral domain and $\mathbf{N} \cong \mathbf{Z}_{3}$ is an $\mathbf{R}$-module. Then the graph $\Gamma\left(\mathbf{R}(+) \mathbf{Z}_{3}\right)$ is a 
Planar.

Proof:

To proof we must note the following two cases to thoughtfulness:

- Case 1: If annihilator of $\mathbf{Z}_{3}$ is equal zero, then $\Gamma\left(\mathbf{R}(+) \mathbf{Z}_{3}\right)$ is equal to $\{(0,1),(0,2)\}$ that is a Planar graph.

- Case 2: If annihilator of $\mathbf{Z}_{3}$ is not equal zero, then graph $\Gamma\left(\mathbf{R}(+) \mathbf{Z}_{3}\right)$ is equal to $\left\{(0,1),(0,2),\left(r_{i}, 0\right),\left(r_{i}, 1\right),\left(r_{i}, 2\right) \quad: \quad r_{i} \in\right.$ $\left.\operatorname{ann}\left(\mathbf{Z}_{3}\right)\right\}$. So, that is a Planar graph.

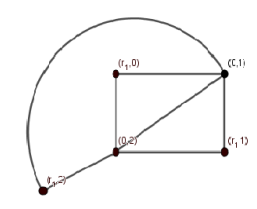

Figure 1: A graph which is a Planar graph.

We begin with the following lemma can be found in [7] to discus the case $\mathbf{N}$ of order 4 .

\section{Lemma 2:}

If the graph $\mathbf{G}$ is a 3-connected planar, then there is a cycle through any five vertices of the graph $\mathbf{G}$.

\section{Theorem 3:}

Suppose that $\mathbf{R}$ is an integral domain and $|\mathbf{N}|=4$ is an $\mathbf{R}$-module. Then we have the following cases:

- Case 1. If the order of $\mathbf{N}$ is equal 4 and annihilator of $N$ is equal to zero, then the graph $\Gamma(\mathbf{R}(+) \mathbf{N})$ is a Planar.

- Case 2. If the order of $\mathbf{N}$ is equal 4 and annihilator of $N$ is not equal to zero, then the graph $\Gamma(\mathbf{R}(+) \mathbf{N})$ is not a Planar.

\section{Proof:}

To proof must note two cases to thoughtfulness:

- Case 1. If the order of $\mathbf{N}$ is equal 4 and annihilator of $N$ is equal to zero, then the graph $\Gamma(\mathbf{R}(+) \mathbf{N})$ is equal to $\left\{\left(0, l_{1}\right),\left(0, l_{2}\right),\left(0, l_{3}\right)\right.$ : $\left.l_{i} \in \mathbf{N}\right\}$. That is a Planar graph.
- Case 2. If the order of $\mathbf{N}$ is equal 4 and annihilator of $N$ is not equal to zero, then the graph $\Gamma(\mathbf{R}(+) \mathbf{N})=\left\{\left(r_{i}, l_{i}\right),\left(0, l_{1}\right),\left(0, l_{2}\right),\left(0, l_{3}\right):\right.$ $\left.l_{i} \in \mathbf{N}, r_{i} \in \operatorname{ann}(\mathbf{N})\right\}$, by previous lemma then the graph is not a Planar graph.

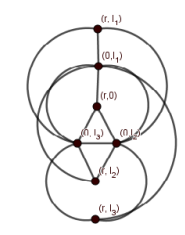

Figure 2: A graph which is not a Planar graph.

The next theorem will discuss when the order of $\mathbf{N}$ is greater than or equal 5 .

\section{Theorem 4:}

Suppose that $\mathbf{R}$ is an integral domain and $|\mathbf{N}| \geq 5$ is an $\mathbf{R}$-module. Then we have the following cases:

- Case 1. If the order of $\mathbf{N}$ is equal to 5 and annihilator of $N$ is equal to zero, then the graph $\Gamma(\mathbf{R}(+) \mathbf{N})$ is a Planar.

- Case 2. If the order of $\mathbf{N}$ is equal to 5 and annihilator of $N$ is not equal to zero, then the graph $\Gamma(\mathbf{R}(+) \mathbf{N})$ is not a Planar.

- Case 3. If the order of $\mathbf{N}$ is greater than 5 , then the graph $\Gamma(\mathbf{R}(+) \mathbf{N})$ is not a Planar.

\section{Proof:}

To proof must note two cases to thoughtfulness:

- Case 1. If the order of $\mathbf{N}$ is equal 5 and annihilator of $N$ is equal zero, then the graph $\Gamma(\mathbf{R}(+) \mathbf{N})$ is equal to $\left\{\left(0, l_{1}\right),\left(0, l_{2}\right),\left(0, l_{3}\right),\left(0, l_{4}\right): l_{i} \in\right.$ $\mathbf{N}\}$. That is a Planar graph.

- Case 2. If the order of $\mathbf{N}$ is equal 5 and annihilator of $N$ is not equal zero, then the graph $\Gamma(\mathbf{R}(+) \mathbf{N})=$ $\left\{\left(r_{i}, l_{i}\right),\left(0, l_{1}\right),\left(0, l_{2}\right),\left(0, l_{3}\right),\left(0, l_{4}\right) \quad: \quad l_{i} \in\right.$ $\left.\mathbf{N}, r_{i} \in \operatorname{ann}(\mathbf{N})\right\}$ has an induced subgraph isomorphic to $K_{5}$. That is not a Planar graph. 


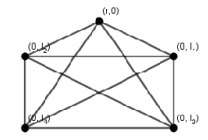

Figure 3: A graph which is not a Planar graph.

- Case 3. If the order of $\mathbf{N}$ is greater than 5, then graph $\Gamma(\mathbf{R}(+) \mathbf{N})$ is equal to $\quad\left\{\left(0, l_{1}\right),\left(0, l_{2}\right),\left(0, l_{3}\right),\left(0, l_{4}\right),\left(0, l_{5}\right), \ldots\right.$, $\left.\left(0, l_{i}\right): l_{i} \in \mathbf{N}\right\}$. That has an induced subgraph isomorphic to $K_{5}$. So, the graph is not a Planar.

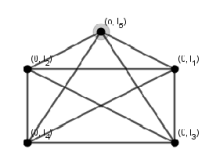

Figure 4: A graph which is not a Planar graph.

\section{When $\Gamma\left(\mathbf{Z}_{n}(+) \mathbf{Z}_{m}\right)$ is Planar grah?}

In this section, we consider the planar for the zerodivisor graph of the idealization ring $\mathbf{Z}_{n}(+) \mathbf{Z}_{m}$, $\Gamma\left(\mathbf{Z}_{n}(+) \mathbf{Z}_{m}\right)$ where $\mathbf{Z}_{m}$ be $\mathbf{Z}_{n}$-module.

Al-Labdi [1], she classified the zero-divisor graph of the idealization ring $\mathbf{Z}_{n}(+) \mathbf{Z}_{m}$.

We begin with the following lemma, when $n$ is a prime number such that $n=p^{\alpha}$ and $m=p$.

\section{Lemma 3:}

Let $n=p^{\alpha}$ and $m=p$ where $p$ is a prime number. Then the graph $\Gamma\left(\mathbf{Z}_{n}(+) \mathbf{Z}_{m}\right)$ have the following cases:

Case 1: If $n$ is equal 4 and $m$ is equal 2, then the graph $\Gamma\left(\mathbf{Z}_{4}(+) \mathbf{Z}_{2}\right)$ is a Planar.
Case 2: If $n$ is equal $p^{\alpha}$ and $m$ is equal $p$ where $p$ is a prime number, $\alpha \geq 3$, then the graph $\Gamma\left(\mathbf{Z}_{p^{\alpha}}(+) \mathbf{Z}_{p}\right)$ is not a Planar.

\section{Proof:}

We consider two cases to proof:

Case 1: If $n$ is equal 4 and $m$ is equal 2, then graph $\Gamma\left(\mathbf{Z}_{4}(+) \mathbf{Z}_{2}\right)$ is equal to $\{(0,1),(2,0),(2,1)\}$. So, that the graph is a Planar.

Case 2: If $n$ is equal $p^{\alpha}$ and $m$ is equal $p$ where $p$ is a prime number greater than 2, $\alpha \geq 3$, then the graph $\Gamma\left(\mathbf{Z}_{p^{\alpha}}(+) \mathbf{Z}_{p}\right)$ is equal $\{(0,1),(0,2), \ldots,(0, p-1),(k p, 0), \ldots,(k p, p-$ $1): k \in\}$. So, it has an induced subgraph $K_{5}$ that is not a Planar graph.

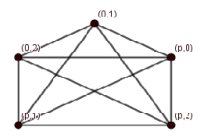

Figure 5: A graph which is not a Planar graph.

\section{Theorem 5:}

Let $m$ is a product of powers of prime numbers $m=p_{1}^{k_{1}} \times p_{2}{ }^{k_{2}} \times \ldots \times p_{l}^{k_{l}}$ and $n$ is product power of primes $n=p_{1}{ }^{s_{1}} \times p_{2}{ }^{s_{2}} \times \ldots \times p_{r}{ }^{s_{r}}$ where $p_{i}$ is a prime number and $l \leq r$. Then the graph $\Gamma\left(\mathbf{Z}_{n}(+) \mathbf{Z}_{m}\right)$ is not a Planar graph.

\section{Proof}

We consider two cases to proof:

If $m$ is product power of primes $m=$ $p_{1}{ }^{k_{1}} \times p_{2}{ }^{k_{2}} \times \ldots \times p_{l}^{k_{l}}$ and $n$ is product power of primes $n=p_{1}{ }^{s_{1}} \times p_{2}{ }^{s_{2}} \times \ldots \times p_{r}{ }^{s_{r}}$ where $p_{i}$ is a prime number and $l \leq r$. Then the graph $\Gamma\left(Z_{p_{1} s_{1} \times p_{2} s_{2} \times \ldots \times p_{r} s_{r}} \quad(+) Z_{p_{1} k_{1} \times p_{2} k_{2} \times \ldots \times p_{l} k_{l}}\right)$ is equal to $\left\{\left(0, h_{i}\right),\left(b_{i}, h_{i}\right): b_{i} \in n h_{i} \in m\right\}$ such that $\operatorname{gcd}\left(b_{i}, n\right) \neq 1$ or $\operatorname{gcd}\left(b_{i}, m\right) \neq 1$. So, it has an induced subgraph $K_{5}$ that is not a Planar graph. 


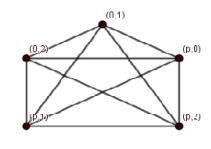

Figure 6: A graph which is not a Planar graph.

\section{Outcome and questions}

In this article, we classify the planarity for the graph of idealization $\Gamma(\mathbf{R}(+) \mathbf{N})$, we conclude in the following theorem.

\section{Theorem 6:}

Let $\mathbf{R}(+) \mathbf{N}$ be an idealization ring. Then the graph $\Gamma(\mathbf{R}(+) \mathbf{N})$ is a Planar graph if the ring $\mathbf{R}$ is an integral domain and the order of $\mathbf{N}$ is less than or equal 4 with $\operatorname{ann}(\mathbf{N})=0$, or the order of $\mathbf{N}$ is equal to 5 with $\operatorname{ann}(\mathbf{N})=0$ and the graph $\Gamma\left(\mathbf{Z}_{n}(+) \mathbf{Z}_{m}\right)$ is a Planar when $n=4, m=2$.

One can ask the following questions:

(1) When the graph $\Gamma(\mathbf{R}(+) \mathbf{N})$ are Eulerian graph?

(2) When the complement graph of idealization ring $\Gamma(\mathbf{R}(+) \mathbf{N})$ are Planar graph?
(3) What is the matching number of the graph $\Gamma(\mathbf{R}(+) \mathbf{N})$ ?

Possible engineering applications of this study can be found in problems of [8] and [9].

\section{References:}

[1] M. Allabadi M, Futher results on the diameter of zero-divisor graphs of some special idealizations, International Journal of Algebra, Vol. 12 (2010), pp. 609-614.

[2] M. Allabadi, On the Diameter of ZeroDivisor Graphs of Idealizations with Respect to Integral Domain, Jordan Journal of Mathematics and Statistics, Vol. 3 (2010), pp. 127-131.

[3] DD. Anderson, M. Naseer, Beck's coloring of a commutative ring, J. Algebra Vol.159 (1993), pp. 500-514.

[4] DF. Anderson, PS. Livingston, The zerodivisor graph of a commutative, J. Algebra, Vol.217 (1999), pp. 434-447.

[5] M. Axtell, J. Stickle, The zero-divisor graph of a commutative rings, textitJornal of Pure and Applied Algebra, Vol.204 (2006), pp. 235-243.

[6] I.Beck, Coloring of a commutative ring, $J$. Algebra, Vol. 116 (1988), pp. 208-226.

[7] B. Jackson, Longest cycles in 3-connected cubic, J. Combin. Theory Ser B, Vol. 41 (1986), pp. 17-26.

[8] N. Boonsim, Racing Bib Number Localization on Complex Backgrounds, WSEAS Transactions on Systems and Control, Vol.13 (2018), pp. 226-231.

[9] T. Ashkan Tashk, H.Jurgen, Esmaeil Nadimi, Automatic Segmentation of Colorectal Polyps based on a Novel and Innovative Convolutional Neural Network Approach, WSEAS Transactions on Systems and Control, Vpl.14 (2019), pp. 384-391.

\section{Creative Commons Attribution License 4.0 (Attribution 4.0 International, CC BY 4.0)}

This article is published under the terms of the Creative Commons Attribution License 4.0 https://creativecommons.org/licenses/by/4.0/deed.en_US 